\title{
Approximation by a composition of Chlodowsky operators and Százs-Durrmeyer operators on weighted spaces
}

\author{
Aydın İzgi
}

Abstract

In this paper we deal with the operators

$$
Z_{n}(f ; x)=\frac{n}{b_{n}} \sum_{k=0}^{n} p_{n, k}\left(\frac{x}{b_{n}}\right) \int_{0}^{\infty} s_{n, k}\left(\frac{t}{b_{n}}\right) f(t) d t, \quad 0 \leqslant x \leqslant b_{n}
$$

and study some basic properties of these operators where $p_{n, k}(u)=\left(\begin{array}{l}n \\ k\end{array}\right) u^{k}(1-u)^{n-k}$, $(0 \leqslant k \leqslant n), u \in[0,1]$ and $s_{n, k}(u)=e^{-n u}(n u)^{k} / k !, u \in[0, \infty)$. Also, we establish the order of approximation by using weighted modulus of continuity.

\section{Introduction}

Let $\rho$ be a polynomial or exponential function such that continuous, monotonically increasing growths to infinity on $[0, \infty)$ satisfy the condition $\rho(x) \geqslant 1$. Classes of functions which satisfy the condition $|f(x)| \leqslant M_{f} \times \rho(x)$ with the norm $\|f\|_{\rho}=\sup _{x \in[0, \infty)}|f(x)| / \rho(x)$ are said to be weighted spaces. There are many studies on approximation on weighted spaces. For instance Ditzian [6] used polynomial weight for Baskakov operators and exponential weight for SzászMirakjan operators. May [22], proved local equivalence theorems on shrinking intervals, using the more general 'operator of exponential type' of which the Baskakov and the Szász-Mirakjan operators are special examples. Lešniewicz and Rempulska [21] introduced some linear positive operators of the Szász-Mirakjan type in the space of continuous functions of one and two variables, having exponential growth at infinity. Gadjiev $[\mathbf{1 0}, \mathbf{1 1}]$ defined the weight spaces $C_{\rho}$ and $B_{\rho}$ of real functions defined on the real axis and showed that Korovkin's theorem in general does not hold on these spaces. Here

$$
B_{\rho}:=\left\{f:|f| \leqslant M_{f} \rho, \rho \geqslant 1 \text { and } \rho \text { is unbounded }\right\}
$$

and

$$
C_{\rho}:=\left\{f \in B_{\rho} \mid f \text { continuous }\right\}
$$

are spaces of functions which are defined on unbounded sets. However, in [10] and [11] it has been shown that this theorem holds on a common subspaces of $C_{\rho}$ and $B_{\rho}$. Coskun [4] proved that a theorem of Korovkin type does not hold on the spaces $C_{\rho_{1}}$ and $B_{\rho_{2}}$ with different weights $\rho_{1}$ and $\rho_{2}$, respectively. Later, in [5], Coskun showed that by some appropriate conditions on the weight functions it holds. Ispir [19] studied modified Baskakov operators on weighted spaces by means of Korovkin's theorems, proved by Gadjiev. We can refer the interested readers to other studies which investigated weight approximation problems for well-known linear positive operators (see, e.g., $[3,7, \mathbf{1 2}, \mathbf{1 3}, \mathbf{1 6}, \mathbf{2 0}, \mathbf{2 4}]$ ) and for general positive linear operators (see, e.g., $[4,8,10,11])$ on infinite intervals. 


\section{Preliminaries}

İbikli and Karslı[17] defined integral modifications of Bernstein-Chlodowsky operators by means of Durrmeyer so as to approximate functions of bounded variation on the interval $\left[0, b_{n}\right]$ which grows to positive semi-axis where $n \rightarrow \infty$. İbikli and others [18] studied these operators for Lebesgue $L_{p}$-integrable functions on the interval $\left[0, b_{n}\right]$. Mazhar and Totik [23] introduced the integral modification of the Szász-Mirakjan operators to approximate functions defined on $[0, \infty)$. These operators and their modifications have been studied by many authors (see, e.g., $[14,15])$.

This study combines Chlodowsky operators and Szász-Durrmeyer operators.

Bernstein-Durrmeyer operators [9] are given by

$$
M_{n}(f ; x)=(n+1) \sum_{k=0}^{n} p_{n, k}(x) \int_{0}^{1} p_{n, k}(t) f(t) d t
$$

where

$$
p_{n, k}(u)=\left\{\begin{array}{l}
\left(\begin{array}{l}
n \\
k
\end{array}\right) u^{k}(1-u)^{n-k}, 0 \leqslant k \leqslant n, \\
0, k>n .
\end{array}\right.
$$

Szász-Durrmeyer operators [23] are given by

$$
S(f ; x)=n \sum_{k=0}^{\infty} s_{n, k}(x) \int_{0}^{\infty} s_{n, k}(t) f(t) d t
$$

where $s_{n, k}(x)=e^{-n x}(n x)^{k} / k !, x \in[0, \infty)$.

Chlodowsky $[\mathbf{3}]$ operators are given by

$$
C_{n}(f ; x)=\sum_{k=0}^{n} p_{n, k}\left(\frac{x}{b_{n}}\right) f\left(\frac{k}{n} b_{n}\right), \quad 0 \leqslant x \leqslant b_{n}
$$

where $\left(b_{n}\right)$ is a positive and increasing sequence with properties $\lim _{n \rightarrow \infty} b_{n}=\infty$ and $\lim _{n \rightarrow \infty} b_{n} / n=0$.

In this study we define the following operators which are combinations of (3) and (2):

$$
Z_{n}(f ; x)=\frac{n}{b_{n}} \sum_{k=0}^{n} p_{n, k}\left(\frac{x}{b_{n}}\right) \int_{0}^{\infty} s_{n, k}\left(\frac{t}{b_{n}}\right) f(t) d t, \quad 0 \leqslant x \leqslant b_{n}
$$

where $\left(b_{n}\right)$ is given in (3) with the difference (see [16])

$$
\lim _{n \rightarrow \infty} \frac{b_{n}^{2}}{n}=0 .
$$

We use the weighted Korovkin's type theorems, proved by Gadjiev [10, 11], and we use the same notation as in $[\mathbf{1 0}]$.

Let $\rho(x)=1+x^{2}, x \in(-\infty, \infty)$ and $B_{\rho}$ be the set of all functions $f$ defined on the real axis satisfying the condition

$$
|f(x)| \leqslant M_{f} \rho(x)
$$

where $M_{f}$ is a constant depending only on $f$. Here $B_{\rho}$ is the normed space with the norm

$$
\|f\|_{\rho}=\sup _{x \in(-\infty, \infty)} \frac{|f(x)|}{\rho(x)}, \quad f \in B_{\rho} .
$$

Here $C_{\rho}$ denotes the subspace of all continuous functions in $B_{\rho}$ and $C_{\rho}^{k}$ denotes the subspace of all functions $f \in C_{\rho}$ with

$$
\lim _{|x| \rightarrow \infty} \frac{|f(x)|}{\rho(x)}=K_{f}<\infty
$$

where $K_{f}$ is a constant depending only on $f$. 
Theorem A $[\mathbf{1 0}, \mathbf{1 1}]$. Let $\left\{T_{n}\right\}$ be a sequence of linear positive operators taking $C_{\rho}$ into $B_{\rho}$ and satisfying the conditions

$$
\lim _{n \rightarrow \infty}\left\|T_{n}\left(t^{v} ; x\right)-x^{v}\right\|_{\rho}=0, \quad v=0,1,2 .
$$

Then for any $f \in C_{\rho}^{k}$,

$$
\lim _{n \rightarrow \infty}\left\|T_{n} f-f\right\|_{\rho}=0
$$

and there exist a function $g \in C_{\rho} \backslash C_{\rho}^{k}$ such that

$$
\lim _{n \rightarrow \infty}\left\|T_{n} g-g\right\|_{\rho} \geqslant 1 .
$$

Applying Theorem A to the operators

$$
T_{n}(f ; x)= \begin{cases}V_{n}(f ; x) & \text { if } x \in\left[0, a_{n}\right] \\ f(x) & \text { if } x>a_{n}\end{cases}
$$

then gives the following theorem.

Theorem B [12]. Let $\left(a_{n}\right)$ be a sequence with $\lim _{n \rightarrow \infty} a_{n}=\infty$ and $\left\{V_{n}\right\}$ be a sequence of linear positive operators mapping $C_{\rho}\left[0, a_{n}\right]$ into $B_{\rho}\left[0, a_{n}\right]$.

If for $v=0,1,2$

$$
\lim _{n \rightarrow \infty}\left\|V_{n}\left(t^{v} ; x\right)-x^{v}\right\|_{\rho,\left[0, a_{n}\right]}=0,
$$

then for any $f \in C_{\rho}^{k}\left[0, a_{n}\right]$ we have

$$
\lim _{n \rightarrow \infty}\left\|V_{n} f-f\right\|_{\rho,\left[0, a_{n}\right]}=0,
$$

where $B_{\rho}\left[0, a_{n}\right], C_{\rho}\left[0, a_{n}\right]$ and $C_{\rho}^{k}\left[0, a_{n}\right]$ denote the same as $B_{\rho}, C_{\rho}$ and $C_{\rho}^{k}$, respectively, but the functions are taken on $\left[0, a_{n}\right]$ instead of $\mathbb{R}$ and the norm is taken as

$$
\|f\|_{\rho,\left[0, a_{n}\right]}=\sup _{x \in\left[0, a_{n}\right]} \frac{|f(x)|}{\rho(x)} .
$$

\section{Auxiliary results}

In this section we give some properties of $Z_{n}(f ; x)$ which we use for the main theorems and an approximation theorem by means of Korovkin's theorem.

If we use properties of the derivative and Leibniz formula, it will be easy to see the following equality for $Z_{n}(f ; x)$ :

$$
Z_{n}\left(t^{p} ; x\right)=\left(\frac{b_{n}}{n}\right)^{p} \sum_{i=0}^{p}\left(\begin{array}{l}
p \\
i
\end{array}\right) \frac{p !}{i !} \frac{n !}{(n-i) !}\left(\frac{x}{b_{n}}\right)^{i} .
$$

Now we will give some special cases of (6) for some $p$ :

$$
\begin{gathered}
Z_{n}(1 ; x)=1 \\
Z_{n}(t ; x)=x+\frac{b_{n}}{n} \\
Z_{n}\left(t^{2} ; x\right)=x^{2}+\frac{x\left(4 b_{n}-x\right)}{n}+\frac{2 b_{n}^{2}}{n^{2}} \\
Z_{n}\left(t^{3} ; x\right)=x^{3}+\frac{x\left[18 b_{n}^{2}+9(n-1) b_{n} x-(3 n-2) x^{2}\right]}{n^{2}}+\frac{6 b_{n}^{3}}{n^{3}} \\
Z_{n}\left(t^{4} ; x\right)=x^{4}+\frac{x}{n^{3}}\left[96 b_{n}^{3}+72(n-1) b_{n}^{2} x\right. \\
\left.+16(n-1)(n-2) b_{n} x^{2}-\left(6 n^{2}-11 n+6\right) x^{3}\right]+\frac{24 b_{n}^{4}}{n^{4}} .
\end{gathered}
$$


Lemma 1. We have

$$
\begin{aligned}
Z_{n, m}(x):= & Z_{n}\left((t-x)^{m} ; x\right)=\left(\frac{b_{n}}{n}\right)^{m} \sum_{j=0}^{m}\left(\begin{array}{l}
m \\
j
\end{array}\right)(-1)^{j} n^{j} \\
& \times \sum_{i=0}^{m-j}\left(\begin{array}{l}
m-j \\
i
\end{array}\right) \frac{(m-j) !}{i !} \frac{n !}{(n-i) !}\left(\frac{n x}{b_{n}}\right)^{i+j} .
\end{aligned}
$$

Proof. It is easy to see the proof by the using the equality

$$
(t-x)^{m}=\sum_{j=0}^{m}\left(\begin{array}{l}
m \\
j
\end{array}\right)(-1)^{j} x^{j} t^{m-j}
$$

and linearity of the operator $Z_{n}$.

By simple calculations, we get

$$
\begin{gathered}
Z_{n, 1}((t-x) ; x)=\frac{b_{n}}{n} \\
Z_{n, 2}(x)=Z_{n}\left((t-x)^{2} ; x\right)=\frac{x\left[2 b_{n}-x\right]}{n}+\frac{2 b_{n}^{2}}{n^{2}} \\
Z_{n, 2}(x) \leqslant \frac{2\left(n x+b_{n}\right) b_{n}}{n^{2}} \\
Z_{n, 4}(x)=Z_{n}\left((t-x)^{4} ; x\right)=\frac{x}{n^{3}}\left[3(n-2) x^{3}-4(n-8) b_{n} x^{2}\right. \\
\left.-12(6 n-1) b_{n}^{2} x+72 b_{n}^{3}\right]+\frac{24 b_{n}^{4}}{n^{4}} \\
\sup _{x \in\left[0, b_{n}\right]} Z_{n, 2}(x) \leqslant \frac{3 b_{n}^{2}}{n} \\
\sup _{x \in\left[0, b_{n}\right]} Z_{n, 4}(x) \leqslant \frac{3(n+47) b_{n}^{4}}{n^{3}} \leqslant 144\left(\frac{b_{n}^{2}}{n}\right)^{2} .
\end{gathered}
$$

4. Approximation of $Z_{n}(f ; x)$ in weighted spaces

Let $\left(b_{n}\right)$ is be a sequence has positive terms, increasing and having the following conditions,

$$
\lim _{n \rightarrow \infty} b_{n}=\infty \quad \text { and } \quad \lim _{n \rightarrow \infty} \frac{b_{n}^{2}}{n}=0 .
$$

Lemma 2. The sequence $\left\{Z_{n}\right\}$ defined by (4) is a sequence of linear positive operators taking $C_{\rho}\left[0, b_{n}\right]$ into $B_{\rho}\left[0, b_{n}\right]$.

Proof. In order to prove this lemma, it is enough to prove $\lim _{n \rightarrow \infty} Z_{n}(\rho(t) ; x)=\rho(x)$ uniformly on $\left[0, b_{n}\right]$ since $\rho(x) \in C_{\rho}\left[0, b_{n}\right]$. By using (7) and (9), we have

$$
Z_{n}(\rho(t) ; x)=\rho(x)+\frac{x\left(4 b_{n}-x\right)}{n}+\frac{2 b_{n}^{2}}{n^{2}} .
$$

Therefore, $\left\|Z_{n}(f ; x)\right\|_{\rho,\left[0, b_{n}\right]}$ is uniformly bounded on $\left[0, b_{n}\right]$ because of

$$
\lim _{n \rightarrow \infty} \sup _{x \in\left[0, b_{n}\right]} \frac{4 b_{n}^{2}}{n}+\frac{2 b_{n}^{2}}{n^{2}}=0
$$

under condition (18). The proof is complete. 
Theorem 1. Let $f \in C_{\rho}^{k}[0, \infty)$. Then

$$
\lim _{n \rightarrow \infty}\left\|Z_{n} f-f\right\|_{\rho,\left[0, b_{n}\right]}=0 .
$$

Proof. Using (7)-(9), we have

$$
\begin{aligned}
\lim _{n \rightarrow \infty}\left\|Z_{n}(1 ; x)-1\right\|_{\rho,\left(0, b_{n}\right]} & =0, \\
\lim _{n \rightarrow \infty}\left\|Z_{n}(t ; x)-x\right\|_{\rho,\left[0, b_{n}\right]} & =\lim _{n \rightarrow \infty} \sup _{x \in\left[0, b_{n}\right]}\left|\frac{b_{n}}{n} \frac{1}{1+x^{2}}\right| \\
& =\lim _{n \rightarrow \infty} \frac{b_{n}}{n}=0, \\
\lim _{n \rightarrow \infty}\left\|Z_{n}\left(t^{2} ; x\right)-x^{2}\right\|_{\rho,\left[0, b_{n}\right]} & =\lim _{n \rightarrow \infty} \sup _{x \in\left[0, b_{n}\right]}\left|\left(\frac{x\left(4 b_{n}-x\right)}{n}+\frac{2 b_{n}^{2}}{n^{2}}\right) \frac{1}{1+x^{2}}\right| \\
& =\lim _{n \rightarrow \infty} \frac{2\left(n+b_{n}\right) b_{n}}{n^{2}}=0 .
\end{aligned}
$$

According to Theorem B, the proof is complete.

\section{Rate of approximation of $Z_{n}(f ; x)$ in weighted spaces}

Now we want to find rate of approximation of the sequence of linear positive operators $\left\{Z_{n}\right\}$ for $f \in C_{\rho}^{k}\left[0, b_{n}\right]$. It is well known that the first modulus of continuity

$$
\omega(f ; \delta)=\sup \{|f(t)-f(x)|: t, x \in[a, b],|t-x| \leqslant \delta\}
$$

does not tend to zero, as $\delta \rightarrow 0$, on any infinite interval.

Ispir [19] defined a weighted modulus of continuity $\Omega_{n}(f ; \delta)$ which tends to zero, as $\delta \rightarrow 0$, on an infinite interval. A similar definition can be found in $[\mathbf{1}]$.

For each $f \in C_{\rho}^{k}\left[0, b_{n}\right]$ given by

$$
\Delta_{n}(f ; \delta)=\sup _{\substack{|h| \leqslant \delta \\ x \in\left[0, b_{n}\right]}} \frac{|f(x+h)-f(x)|}{\left(1+x^{2}\right)\left(1+h^{2}\right)} .
$$

In [19] Ispir showed the following properties of $\Delta_{n}(f ; \delta)$ :

(i) $\lim _{\delta \rightarrow 0} \Delta n(f ; \delta)=0$ for every $f \in C_{\rho}^{k}\left[0, b_{n}\right]$;

(ii) for every $f \in C_{\rho}^{k}\left[0, b_{n}\right]$ and $t, x \in\left[0, b_{n}\right]$,

$$
|f(t)-f(x)| \leqslant 2\left(1+\delta_{n}^{2}\right)\left(1+x^{2}\right) \Delta_{n}\left(f ; \delta_{n}\right) . S_{n}(t, x),
$$

where

$$
S_{n}(t, x)=\left(1+\frac{|t-x|}{\delta_{n}}\right)\left(1+(t-x)^{2}\right)
$$

It is easy to see that

$$
S_{n}(t, x) \leqslant \begin{cases}2\left(1+\delta_{n}^{2}\right) & \text { if }|t-x| \leqslant \delta_{n} \\ 2\left(1+\delta_{n}^{2}\right) \frac{(t-x)^{4}}{\delta_{n}^{4}} & \text { if }|t-x| \geqslant \delta_{n} .\end{cases}
$$

Theorem 2. Let $f \in C_{\rho}^{k}\left[0, b_{n}\right]$. Then for all $n$ sufficiently large,

$$
\left\|Z_{n} f-f\right\|_{\rho,\left[0, b_{n}\right]} \leqslant K . \Delta_{n}\left(f ; \sqrt{\frac{b_{n}^{2}}{n}}\right) .
$$

where $K$ is a positive constant. 
Proof. If we use (7) and the properties of linear positive operators $Z_{n}$

$$
\begin{aligned}
\left|Z_{n}(f ; x)-f(x)\right| & \leqslant Z_{n}(|f(t)-f(x)| ; x) \\
& \leqslant 2\left(1+\delta_{n}^{2}\right)\left(1+x^{2}\right) \Delta_{n}\left(f ; \delta_{n}\right) Z_{n}\left(S_{n}(t, x) ; x\right) .
\end{aligned}
$$

By (20) we get

$$
S_{n}(t, x) \leqslant 2\left(1+\delta_{n}^{2}\right)\left[1+\frac{(t-x)^{4}}{\delta_{n}^{4}}\right]
$$

for all $x \in\left[0, b_{n}\right], t \in[0, \infty)$. Thus, for $x \in\left[0, b_{n}\right]$ and using (17),

$$
\begin{aligned}
\left|Z_{n}(f ; x)-f(x)\right| & \leqslant 4\left(1+\delta_{n}^{2}\right)^{2}\left(1+x^{2}\right)\left[1+\frac{1}{\delta_{n}^{4}} Z_{n, 4}(x)\right] \Delta_{n}\left(f ; \delta_{n}\right) \\
& \leqslant 4\left(1+\delta_{n}^{2}\right)^{2}\left(1+x^{2}\right)\left[1+\frac{144}{\delta_{n}^{4}}\left(\frac{b_{n}^{2}}{n}\right)^{2}\right] \Delta_{n}\left(f ; \delta_{n}\right) .
\end{aligned}
$$

Set $\delta_{n}=\sqrt{b_{n}^{2} / n}$ and consider $\delta_{n} \leqslant 0.1$ for sufficiently large $n$. Since $\lim _{n \rightarrow \infty} b_{n}^{2} / n=0$, the proof will be complete.

REMARK 1. This kind of theorem is studied for different operators (for instance, SzászMirakjan and Baskakov operators: see $[\mathbf{1 9}, \mathbf{2 0}]$ ) in the norm $\|\cdot\|_{\rho^{3}}$, but our theorem is studied in the norm $\|\cdot\|_{\rho}$. For instance in $[\mathbf{1 9}]$ it has been given in the form of a theorem: if $f \in C_{\rho}^{k}\left[0, b_{n}\right]$. Then the inequality

$$
\sup _{x \in\left[0, b_{n}\right]} \frac{\left|L_{n}(f ; x)-f(x)\right|}{\left(1+x^{2}\right)^{3}} \leqslant K \Delta_{n}\left(f ; b_{n}^{-1 / 4}\right)
$$

holds where the sequence $\left(b_{n}\right)$ is as in $(3)$ and $\left(L_{n}\right)$ is the Baskakov operators sequence $L_{n}(f ; x)=\sum_{k=0}^{\infty} f(k / n) \varphi_{n}^{k}(x)(-x)^{k} / k !, x \in[0, \infty)$.

Thus, Theorem 2 has better order approximation that the analog theorems which were given in $[\mathbf{1 9}, \mathbf{2 0}]$.

\section{A Voronovskaya-type theorem}

In this section, we prove a Voronovskaya-type theorem for the operators $Z_{n}$.

Theorem 3. For every $f \in C_{\rho}^{k}\left[0, b_{n}\right]$ such that $f^{\prime}, f^{\prime \prime} \in C_{\rho}^{k}\left[0, b_{n}\right]$, we have

$$
\lim _{n \rightarrow \infty} \frac{n}{b_{n}}\left\{Z_{n}(f ; x)-f(x)\right\}=f^{\prime}(x)+x f^{\prime \prime}(x)
$$

uniformly with respect to $x \in\left[0, b_{n}\right]$.

Proof. Let $f, f^{\prime}, f^{\prime \prime} \in C_{\rho}^{k}\left[0, b_{n}\right]$. In order to prove the theorem, by Taylor's theorem we write

$$
f(t)= \begin{cases}f(x)+(t-x) f^{\prime}(x)+\frac{1}{2}(t-x)^{2} f^{\prime \prime}(x)+(t-x)^{2} \eta(t-x) & \text { if } t \neq x \\ 0 & \text { if } t=x\end{cases}
$$

where $\eta(h)$ tends to zero for all $h$ converging to zero.

Now from (7), (13) and (14)

$\frac{n}{b_{n}}\left\{Z_{n}(f ; x)-f(x)\right\}=\frac{n}{b_{n}} \frac{b_{n}}{n} f^{\prime}(x)+\frac{1}{2} \frac{n}{b_{n}}\left[\frac{2 b_{n} x}{n}-\frac{x^{2}}{n}+\frac{2 b_{n}^{2}}{n^{2}}\right] f^{\prime \prime}(x)+\frac{n}{b_{n}} Z_{n}\left((t-x)^{2} \eta(t-x) ; x\right)$.

If we apply the Cauchy-Schwarz-Bunyakovsky inequality for $Z_{n}\left((t-x)^{2} \eta(t-x) ; x\right)$, we conclude that

$$
\frac{n}{b_{n}}\left|Z_{n}\left((t-x)^{2} \eta(t-x) ; x\right)\right| \leqslant \sqrt{\frac{n}{b_{n}^{2}} Z_{n}\left((t-x)^{4} ; x\right)} \sqrt{n Z_{n}\left((\eta(t-x))^{2} ; x\right)} .
$$




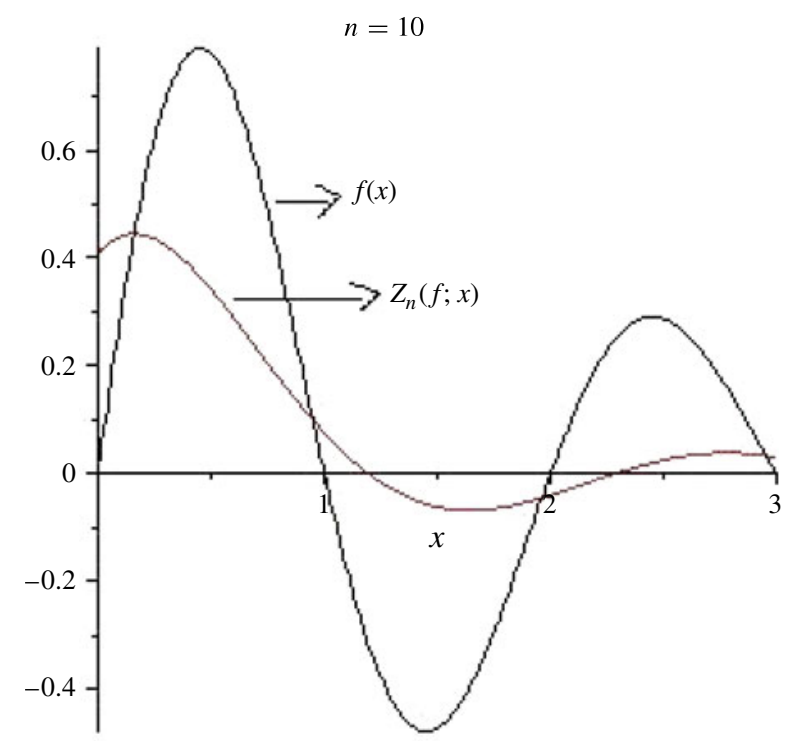

Figure 1. Approximation of $f$ by $Z_{n}(f ; x), n=10$.

If we consider (17) and condition (18) we obtain

$$
\sqrt{\frac{n}{b_{n}^{2}} Z_{n}\left((t-x)^{4} ; x\right)} \leqslant \sqrt{\frac{n}{b_{n}^{2}}\left[144\left(\frac{b_{n}^{2}}{n}\right)^{2}\right]} .
$$

Here $\lim _{n \rightarrow \infty} \sqrt{\left(n / b_{n}^{2}\right) Z_{n}\left((t-x)^{4} ; x\right)}=0$ is bounded with respect to $x \in\left[0, b_{n}\right]$. On the other hand, by assumption $\lim _{t \rightarrow x} \eta(t-x)=0$. So, it follows that

$$
\lim _{n \rightarrow \infty} \frac{n}{b_{n}}\left|Z_{n}\left((t-x)^{2} \eta(t-x) ; x\right)\right|=0 .
$$

Then we have

$$
\frac{n}{b_{n}}\left\{Z_{n}(f ; x)-f(x)\right\}=f^{\prime}(x)+x f^{\prime \prime}(x)-\frac{1}{2} \frac{x^{2}}{b_{n}} f^{\prime \prime}(x)+\frac{b_{n}}{n} f^{\prime \prime}(x) .
$$

If we take lim on both sides with respect to $x \in\left[0, b_{n}\right]$, we will obtain the desired result.

6.1. An example for Theorem 3

Choose $f(x)=x^{3}$. Then $f^{\prime}(x)=3 x^{2}, f^{\prime \prime}(x)=6 x$ and $f^{\prime}(x)+x f^{\prime \prime}(x)=3 x^{2}+6 x^{2}=9 x^{2}$. Now use (10)

$$
\begin{gathered}
\frac{n}{b_{n}}\left\{Z_{n}\left(t^{3} ; x\right)-x^{3}\right\}=\frac{18 b_{n}}{n} x+\frac{9(n-1)}{n} x^{2}-\frac{(3 n-2)}{n b_{n}} x^{3}+\frac{6 b_{n}^{2}}{n^{2}} \\
\lim _{n \rightarrow \infty} \frac{n}{b_{n}}\left\{Z_{n}\left(t^{3} ; x\right)-x^{3}\right\}=9 x^{2}=f^{\prime}(x)+x f^{\prime \prime}(x) .
\end{gathered}
$$

\subsection{Some plots}

Figures 1-4 show plots for the function $f(x)=\sin (\pi x) \cdot \exp (-x / 2)$ on the interval $[0,3]$ for some $n$, generated using Maple14. 


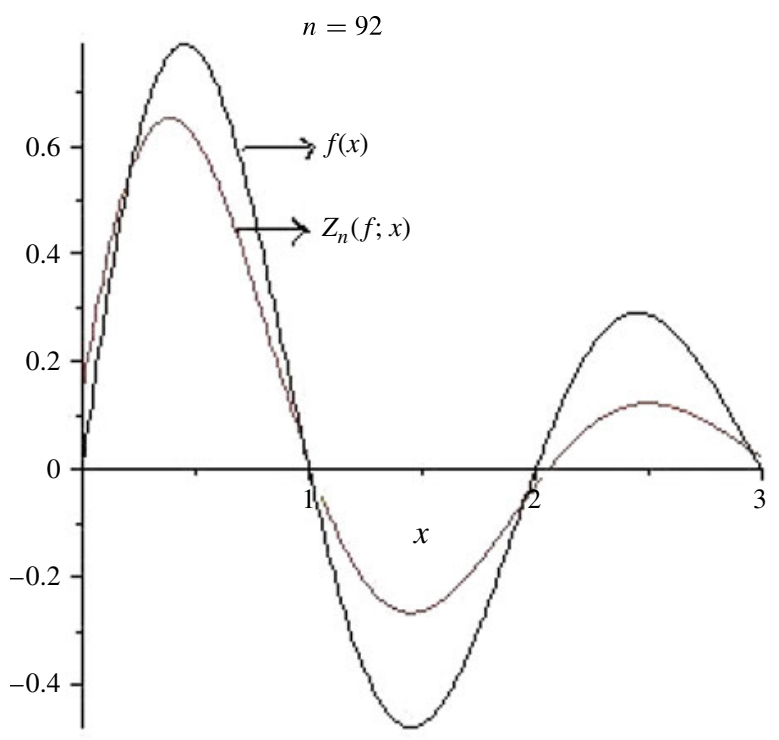

Figure 2. Approximation of $f$ by $Z_{n}(f ; x), n=92$.

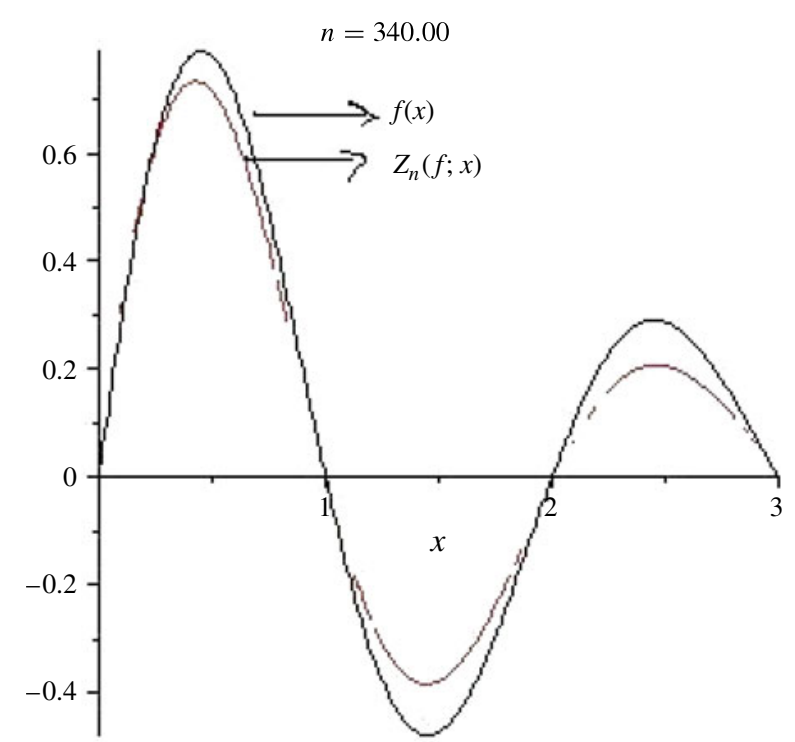

Figure 3. Approximation of $f$ by $Z_{n}(f ; x), n=340$. 


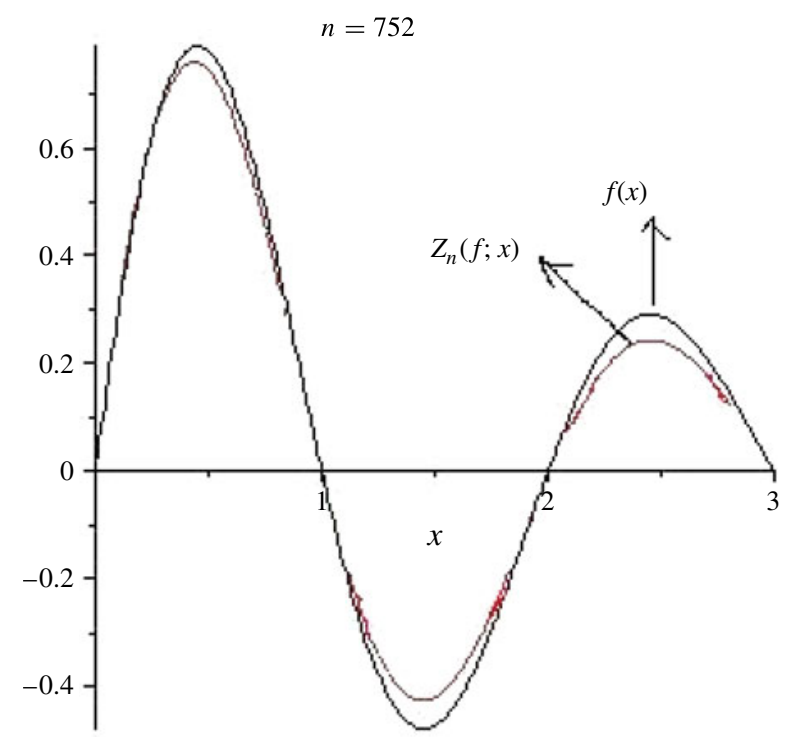

Figure 4. Approximation of $f$ by $Z_{n}(f ; x), n=752$.

\section{References}

1. N. I. ACHIESER, Lectures on the theory of approximation (OGIZ, Moscow-Leningrad, 1947) (Russian); Theory of approximation, translated by C. J. Hymann (Frederick Ungar, New York, 1956) 208-226.

2. M. BECKER, 'Global approximation theorems for Szász-Mirakjan and Baskakov operators in polynomial weight spaces', Indiana Univ. Math. J. (1978) 127-142.

3. I. Chlodowsky, 'Sur le développment des fonctions défines dans un interval infinien séries de polynómes de S. N. Bernstein', Compositio Math. 4 (1937) 380-392.

4. T. CoşKun, 'Some properties of linear positive operators on the spaces of weight functions', Commun. Fac. Sci. Univ. Ank. Sér. A1 47 (1998) 175-181.

5. T. CoşKun, 'Weighted approximation of continuous functions by sequences of linear positive operators', Proc. Indian Acad. Sci. (Math. Sci.) 110 (2000) 357-362.

6. Z. DitZIAn, 'Convergence of sequences of linear positive operators: remarks and applications', J. Approx. Theory 14 (1975) 296-301.

7. Z. Ditzian, 'On global invers theorems of Szăsz and Baskakov operators', Canad. J. Math. 31 (1972) $255-263$.

8. O. DoĞRU, 'Weighted approximation of continuous functions on the all positive axis by modified linear positive operators', Int. J. Comput. Numer. Anal. Appl. 1 (2002) 135-147.

9. J. L. Durrmeyer, 'Une formule d'inversion de la transformee de Laplace: Applications a la theorie de moments', these de 3e cycle, Faculte des sciences de 1'universite de Paris (1967).

10. A. D. Gadzhiev, 'The convergence problem for a sequence of linear operators on unbounded sets and theorem analogous to that of P. P. Korovkin', Soviet Math. Dokl. 15 (1974) 1433-1436.

11. A. D. Gadzhiev, 'Theorems of the type P. P. Korovkin theorems', Math. Zametki 20 (1976) 781-786; English translation in Math. Notes 20 (1976), 996-998.

12. A. D. Gadzhiev, R. O. Efendiev and E. IBIKLI, 'Generalized Bernstein-Chlodowsky polynomials', Rocky Mount. J. Math. 28 (1998) 1267-1277.

13. E. A. Gadzhieva and E. IBIKLI, 'Weighted approximation by Bernstein-Chlodowsky polynomials', Indian J. Pure Appl. Math. 30 (1999) 83-87.

14. V. GuptA, 'Simultaneous approximation by Szăsz-Durrmeyer operators', Math. Student 64 (1995) 27-36.

15. V. Gupta and P. N. Agarval, 'An estimation of the rate of convergence for modified Szăsz-Mirakjan operators of functions of bounded variation', Publ. Inst. Math. (Beograd) (N.S.) 49 (1991) 97-103.

16. E. IBIKLI, 'On approximation by Bernstein-Chlodowsky polynomials', Math. Balkanica (N.S.) 17 (2003) 259-265.

17. E. IBIKLI and H. KARsLi, 'Rate of convergence of Chlodowsky type Durrmeyer operators', J. Inequal. Pure Appl. Math. 6 (2005) no. 4, paper 106.

18. E. Ibikli, A. Izgi and I. Buyukyazici, Approximation of $L^{p}$ - integrable functions by linear positive operators. ICCAM 2006, 10-14 July.

19. N. IspIR, 'On modified Baskakov operators on weighted spaces', Turkish J. Math. 26 (2001) 355-365. 
20. N. Ispir and Ç. Atakut, 'Approximation by modified Szasz-Mirakjan operators on weighted spaces', Proc. Indian Acad. Sci. (Math. Sci.) 112 (2002) 571-578.

21. M. LEŠNIEWICZ and L. R. RempluskA, 'Approximation by some operators of Szasz-Mirakjan type in exponential weight spaces', Glas. Mat. 32 (1997) 57-69.

22. C. P. MAY, 'Saturation and inverse theorems for combinations of class of exponential type operators', Canad. J. Math. 28 (1976) 1224-1250.

23. S. M. Mazhar and V. Totik, 'Approximation by modified Szász operators', Acta Sci. Math. 49 (1985) $257-269$.

24. Z. WALCZAK, 'On certain linear positive operators in exponential weighted spaces', Math. J. Tojama Univ. 25 (2002) 19-118.

\author{
Aydın İzgi \\ Matematik Bölümü \\ Harran Üniversitesi \\ Fen-Edebiyat Fakültesi \\ Osmanbey Kampüsü \\ 63300 Sanliurfa \\ Turkey \\ aydinizgi@yahoo.com, \\ aydinizgi1@gmail.com
}

